\title{
Research Progress of Antibiotic Pollution and Treatment Technologies in China
}

\author{
Wenju Yang ${ }^{1,2}$, Feierkate Fulidong ${ }^{1,2}$, Junwen $\mathrm{Ma}^{1}$, and Yubo Cui ${ }^{1,2, *}$ \\ ${ }^{1}$ Key Laboratory of Biotechnology and Bioresources Utilization, Ministry of Education, Dalian Minzu University, Dalian 116600, China \\ ${ }^{2}$ College of Environment and Resources, Dalian Minzu University, Dalian 116600, China
}

\begin{abstract}
In recent years, antibiotic pollution has become increasingly serious in China, and many kinds of antibiotics have been detected in river basins, soil and drinking water. How to control antibiotic pollution has become a social concern in the process of building ecological civilization. This paper introduces the current situation, sources and harms of antibiotic pollution and analyzes the current development status of antibiotic pollution treatment technologies in China, such as traditional treatment technology, new constructed wetland technology and phytoremediation technology, etc. Some suggestions on antibiotic pollution control were given from the aspects of relevant laws and regulations, the use of antibiotic treatment technology according to local conditions and the combination of multiple processes.
\end{abstract}

\section{Introduction}

With the steady advance of ecological civilization construction, China's environmental protection is gradually on the rise, and the focus of people's attention is gradually shifting from traditional pollutants to new pollutants. In recent years, the emergence of "Superbacteria" has pushed the problem of antibiotic pollution to the forefront and become a hot spot of social concern. At present, the commonly used antibiotics are mostly synthetic or semi-synthetic compounds, mainly including sulfonamides, macrolides, quinolones and tetracyclines. China is a major producer and consumer of antibiotics. It uses 210,000 tons of antibiotics every year, accounting for about $1 / 5$ of the world's total.

\section{Current situation and sources of environmental antibiotic pollution}

\subsection{Current situation of antibiotic pollution in the environment}

In recent years, antibiotics have been detected in surface water, groundwater and even drinking water in China. Generally speaking, the Yangtze river delta, the pearl river delta and the Beijing-Tianjin-Hebei region are far higher than other regions, and the eastern region is far higher than the central and western regions. According to relevant statistics, a number of antibiotics were detected in the Yangtze River, the Yellow River, the Pearl River, the Chaohu Lake and the Huangpu River in China. Many kinds of antibiotics were found in drinking water of Anhui and Jiangsu provinces. A number of antibiotics have also been detected in groundwater in
Anhui, Guangxi and Tianjin ${ }^{[1]}$. With the widespread use of antibiotics, they enter the soil in a variety of ways; There are mainly agricultural and breeding wastes, human and animal wastes applied as fertilizers and expired antibiotics discarded by families ${ }^{[2]}$. The problem of antibiotic pollution has posed a potential threat to the ecological environment and human body.

\subsection{Sources of antibiotics in the environment}

Large-scale, intensive livestock, poultry and aquaculture are one of the main sources of antibiotics in the environment. We against China in 2019 Dalian three at the bottom of the sea cucumber breeding base of water and sediment samples for testing, in the water and sediment samples were detected with levofloxacin, doxycycline and other antibiotics, test results show that the bottom sediment types of antibiotics is rich in water samples, and with the increase of breeding time, the greater the amount of antibiotics enrichment of sediments at the bottom.

Medical wastewater and domestic sewage are another important source of antibiotics in the environment. China is a major user of antibiotics. The prophylactic use of antibiotics in medical treatment leads to the introduction of antibiotics into medical treatment and domestic sewage. At present, the current sewage treatment technology in China is not ideal in the treatment of antibiotics. In addition, pharmaceutical wastewater contains a variety of refractory active antibiotics, which have certain biological toxicity. In the biochemical process of sewage treatment plants, the high concentration of antibiotics in the wastewater will inhibit the growth of microorganisms in the biochemical stage and react to the degradation process of wastewater.

* Corresponding author: cyb@dlnu.edu.cn 


\section{$3 \underset{\text { Harm caused by antibiotic }}{4} \begin{gathered}\text { Antibiotic } \\ \text { technology }\end{gathered}$
contamination}

\subsection{Harm to water ecological environment}

Antibiotic pollution not only changes the biome structure of the water ecological environment, but also destroys the healthy operation of the water ecological environment, and restricts the social and economic development and the sustainable development of water resource utilization ${ }^{[3]}$.Many scholars research results show that antibiotic effects of toxic to aquatic organisms, 226 kinds of antibiotics toxicity test results show that $1 / 5$ of the algae with high toxicity (EC50 (half effect concentration) $<1 \mathrm{mg} / \mathrm{L}$ ), $6 \%$ of the antibiotics on daphnia toxic $($ EC50<0.1 $\mathrm{mg} / \mathrm{L})$, about a third of the antibiotics with high toxicity to fish $(\mathrm{EC} 50<1 \mathrm{mg} / \mathrm{L})$, more than half of all antibiotics for toxic fish $(\mathrm{EC} 50<10 \mathrm{mg} / \mathrm{L})$. Related studies have shown that antibiotics have a variety of effects on plankton in the ocean. On the one hand, low or specific concentrations of antibiotics can promote the growth of low microalgae, on the other hand, they act as inhibitors on the growth of algae ${ }^{[1]}$.

\subsection{Harm to human health}

Long-term consumption of food containing antibiotics will pose a potential threat to human health. Antibiotics accumulate in the food chain and eventually enter the body; In addition, due to the poor removal effect of drinking water treatment technology on antibiotics, antibiotics and their degradation products also enter the human body with drinking water. Low concentrations of antibiotics do not harm to the human body, but can cause allergies and toxic side effects, and may even cause cancer, teratogenic, mutagenic.

\subsection{Induce the bacteria to develop resistance}

When bacteria are exposed to low levels of antibiotics for a long time, they develop resistance. Four kinds of sulfonamine-resistance genes and seven kinds of tetracycline resistance genes were detected in Haihe River water and sediments, and the resistance genes in sediments were 120-2000 times higher than those in water ${ }^{[1]}$. Drug resistance will weaken the growth inhibition effect of antibiotics on bacteria and pose a potential threat to humans and ecosystems.

\subsection{Affect the soil ecological balance}

A variety of antibiotics were detected in the fields around large-scale livestock and poultry farms, with the content ranging from $1.75 \mu \mathrm{g} \cdot \mathrm{kg}^{-1}$ to $2.45 \mathrm{mg} \cdot \mathrm{kg}^{-1[2]}$. Antibiotics are extremely toxic to soil microorganisms, and earthworms are very sensitive to antimicrobial drugs. About 56 components of veterinary drugs are released into the soil, seriously affecting the ecological balance of the soil.

\subsection{Traditional treatment techniques}

\subsubsection{Sludge digestion treatment}

At present, sludge treatment is still widely used for antibiotic removal. The current sewage treatment system is mainly aimed at traditional pollutants, but it is still unable to effectively remove the emerging antibiotics. Because the sewage treatment plant sludge contains a large number of antibiotics, resulting in a certain amount of antibiotics in the water. In recent years, some scholars have studied the digestion of sludge to improve the effective removal rate of antibiotics. The results show that the removal rate under anaerobic condition is higher than that under aerobic condition, and the removal rate increases with the increase of temperature. In the same digestive system, the removal rate increases with the growth of sludge age. Sludge digestion treatment can be used in combination with multiple processes and has a good prospect. However, the removal rate of antibiotics is low, and is accompanied by sludge swelling, single composition and other defects.

\subsubsection{Physical and chemical methods}

The traditional physicochemical methods for antibiotic removal are adsorption, photodegradation and electrolysis. In the adsorption method, the adsorption effect is related to the particle size of the adsorption matrix. The larger the particle size, the better the adsorption effect. Some scholars have found that the removal rate of ampramycin in water environment by electrolysis can reach more than $50 \%{ }^{[4]}$. S.Y. Zhang ${ }^{[5]}$ used activated carbon granules and attapulgite modified with cetyl trimethyl ammonium bromide as adsorbents to remove sulfonamides antibiotics in water. The experimental results showed that the removal rate of sulfonamides antibiotics by activated carbon reached more than $96 \%$, and the attapulgite modified by cetyl trimethylammonium bromide as adsorbent had a selective effect on sulfonamides antibiotics with many influencing factors. Chemical reagent should be added in the later stage of the adsorption method, which is easy to cause secondary pollution and high separation and recovery cost.

\subsubsection{Membrane treatment technology}

The membrane treatment technique is one of the most advanced techniques for antibiotic removal, which has a good effect on antibiotic removal and is negatively correlated with the pore size of the membrane bioreactor. Using activated sludge anoxic-aerobic membrane bioreactor (A/O-MBR) and immobilized anoxic-aerobic membrane bioreactor (I-A/O-MBR) to treat antibioticcontaining wastewater, it was found that SRT and HRT had a great influence on the degradation efficiency of 
antibiotics. A/O-MBR reactor is greatly affected by SRT. When SRT reaches 30d, the removal effect of the reactor on six typical antibiotics can reach more than $84.4 \%$, and the operation of A/O-MBR reactor under longer SRT conditions is less affected by HRT. When the HRT in the $\mathrm{I}-\mathrm{A} / \mathrm{O}-\mathrm{MBR}$ reactor reaches $6 \mathrm{~h}$, the pollutants in the water can fully contact with the microorganisms in the reactor and achieve a better degradation effect. If it is lower than $6 \mathrm{~h}$, the degradation effect of all kinds of pollutants will be reduced ${ }^{[6]}$. The application of membrane bioreactor focuses on the control of SRT and HRT and the guarantee of biomass, the adsorption and re-release of antibiotics and the toxicity of some reaction products.

\subsection{New treatment technologies}

\subsubsection{Constructed wetland technology}

Constructed wetland is a comprehensive ecosystem composed of soil, plants, microorganisms and media, which is artificially constructed, controlled and operated. It mainly uses the multiple synergistic effects of soil, microorganism and plant, and has a good removal effect on common antibiotics. The removal efficiency of antibiotics was greatly affected by the hydraulic retention time, the proportion of plants and the choice of fillers. The removal effect of tetracycline and quinolone antibiotics is not affected by wetland factors, and photolysis is the most likely way to remove tetracycline [7]. Constructed wetland technology, matrix and hydraulic load are the main factors affecting the removal efficiency of sulfonamides antibiotics. Through the influence of different wetland conditions on the removal effect of sulfonamides antibiotics, it was found that the removal effect of some sulfonamides antibiotics was positively correlated with the hydraulic retention time, and the removal effect was the best at $96 \mathrm{~h}^{[8]}$. The study also found that the longer hydraulic retention time and the lower hydraulic load were helpful to improve the removal rate of sulfonamides antibiotics, while other factors had little effect on the removal of sulfonamides antibiotics. Constructed wetland technology has the advantages of low cost, easy to maintain, good effect, abundant microbial population, energy saving and pollution-free, etc., but constructed wetland covers a large area and is not easy to control.

\subsubsection{Ultrasonic degradation technology}

In recent years, ultrasonic degradation is a new water treatment technology which is widely concerned. Ultrasonic degradation technology uses ultrasonic wave to excite the bubble nucleus in the liquid to produce instantaneous high temperature and instantaneous high pressure, thus breaking the chemical bond of antibiotics in the liquid. At the same time, the water vapor generates hydroxyl free radicals under high temperature and high pressure to oxidize organic pollutants in the solution, so that it can be converted into molecules or ions that can be more easily handled. Ultrasonic degradation technology has a broad application prospect, but it only has obvious effect on the degradation of sulfonamides antibiotics, and its high cost and high energy consumption have become important factors restricting the practical application of this technology.

\subsubsection{Low temperature plasma technology}

Low temperature plasma technology is a technology that accelerates the degradation of antibiotics in chemical reaction to achieve the removal effect by taking ozone, electron radiation and ultraviolet light produced by a large number of active particles in the ion as the occurrence conditions. The low temperature plasma technology was used to degrade antibiotics. The antibiotics began to degrade in a short time after the reaction and the degradation rate was fast. In addition, some scholars found that under the synergism of ultraviolet light, electronic radiation and ozone, the degradation effect of antibiotics was much higher than that of each. The low temperature plasma technology can degrade antibiotics effectively, and the operation is simple and convenient without adding any chemical reagents. However, the reaction rate, conditions and amount are difficult to control, and the combined effect and by-products are difficult to estimate ${ }^{[4]}$.

\subsubsection{Soil infiltration technology}

Soil infiltration technology by physical adsorption of soil aquifer settlement, ion exchange and other chemical and biological role of synergistic effect to remove antibiotics in order to make the content of the water pollutants from sewage concentration or reach the level of recycled technology method, the method of water body in a variety of antibiotics has a better removal effect. Relevant studies have shown that the removal rate of some antibiotics by this technique exceeds $95 \%{ }^{[4]}$. However, when the concentration of antibiotics exceeds the environmental carrying capacity, the antibiotics will pollute the local soil and groundwater. The technology is difficult to be applied in practice, so the area of soil percolation should be strictly controlled during the operation of the system to prevent it from polluting the surrounding environment.

\subsubsection{Phytoremediation technology}

Phytoremediation is the use of plant tissues through decomposition, transformation and other metabolic effects to remove antibiotics. Through the experimental study on two aquatic plants, pistia stratiotes and eichhornia crassipes ${ }^{[8]}$, it was found that both of them had certain removal effect on tetracycline hydrochloride, aureomycin hydrochloride, ampicillin penicillin and oxytetracycline hydrochloride, four commonly used antibiotics in the water environment, and could achieve obvious removal effect in a short time. The removal rates of tetracycline hydrochloride and ampicillin of the two plants reached more than $70 \%$, which was obviously better than that of the four antibiotics and the hyacinth 
japonicus. S.J. Zhou et al. ${ }^{[9]}$ studied the removal effect of flower plants on antibiotics. Through the experimental study on four kinds of flower plants, such as copper grass, spider plant, peace lilies and scindapsus, the results showed that all four kinds of plants had certain removal effect on norfloxacin in soil and water, but the removal rate was relatively low. P.C. Zhou et al. ${ }^{[10]}$ found that, by investigating the removal effect of four aquatic plants, such as pennisetum hydridum, canna lily, pickerel weed and umbrella sedge, on antibiotics used in animals, the removal effect of pennisetum hydridum on amoxicillin was better than the other three plants, and the removal effect of canna lily was better than the other two. In the removal experiments of doxycycline hydrochloride and macrolide antibiotics, the removal effect of canna lily was better than that of other three plants. The effect of phytoremediation technology depends on the concentration of antibiotics in the environment and the development degree of the root system of the plant itself.

The technical mechanism of phytoremediation should be the comprehensive effects of direct enrichment or absorption through roots during plant growth, absorption and degradation of root exudates, and degradation of antibiotics by microorganisms in the growing environment to achieve the purpose of repair [11]. Antibiotics inhaled from plants are either broken down or concentrated in their original form.

\section{Conclusions and prospects}

The treatment of antibiotic pollution has become a realistic problem that China needs to face. We urgently need a new and widely used antibiotic pollution control technology. However, the new antibiotic pollution control technologies such as ultrasonic degradation, lowtemperature plasma, and phytoremediation have their own advantages and disadvantages. In the future, we will focus on the following aspects.

(1) Introduce relevant laws and regulations to strictly control the misuse of antibiotics. In particular, to prevent the use of prophylactic antibiotics and excessive administration of antibiotics in the process of breeding.

(2) Implement antibiotic control strategies according to local conditions. Because of the regional characteristics of antibiotic pollution, local environmental departments should implement the "one place, one policy" plan according to the local characteristics of antibiotic pollution.

(3) Accelerating the practical application of antibiotic pollution prevention and control technology. On the basis of relevant theoretical research, it is necessary to apply the theoretical achievements into the engineering construction step by step and also serve as a yardstick to test the theoretical achievements.

(4) Multi-process combination application. In the existing sewage treatment facilities, antibiotics pollution control module is added to explore the optimal combination of traditional and emerging pollutants in the practical application.

\section{Acknowledgements}

The research was financed by the Science and Technology Innovation Foundation of Dalian, China (2018J12SN080) and Natural Science Foundation (National Innovation Joint Fund) of Liaoning, China.

\section{References}

1. G.D. Zhang, W.P. Dong, X.H. Liu. et al. Environ. Chem. Occurrence, fate and risk assessment of antibiotics in water environment of China, 37, 14911500, (2018)

2. S. Chen, F. Xu, W. Zhang, et al. Environ. Chem. Research progress in pollution situation and environmental behavior of Sulfonamides, 38, 15571569, (2019)

3. G.Y. Liu, K. Ge, Z.H. Wang, et al. Journal of Bohai University (Natural Science Edition), Research progress on antibiotic pollution in coastal waters, 38, 331-336, (2017)

4. X.W. Chen, Y.X. Liang, X.F. Yu, et al. Environ. Sci. Technol. The contamination and treatment technologies for antibiotics in water: a review, $\mathbf{4 0 ,}$ 125-132, (2017)

5. S.Y. Zhang, Lanzhou Jiaotong University, Adsorptive Removal of Sulfonamide antibiotics from aqueous solution by Granular Activated Carbon and Modified Attapulgite, (2019)

6. R.Y. Jia, S.Q. Xia, S.F. Zhang, Water Purification Technology, Comparison of Antibiotics Removal Performance and Microbial Community Structures between Two Different MBR Processes, 30, 2833+39, (2011)

7. C.C. Geng, Y.J. Wang, Adm. Technol. Environ. Monit. Research Progress of Biochemical Treatment on Antibiotics Removal from Wastewater, 31, 1216+56, (2019)

8. X.J. Chen, F.Y. Li, Y.B. Hao, Subtropical Plant Science, The Preliminary Exploration of Remediation the Antibiotics Polluted Water by Two Hydrophytes, 41, 1-7, (2012)

9. S.J. Zhou, F.Q. Feng, Guizhou Agricultural Sciences, Rehabilitation of Soil and Water Contaminated by Expired Norfloxacin Capsules by Four Flowers, 47, 114-117, (2019)

10. P.C. Zhou, X.Q. Liu, X.S. Kang, et al. Journal of South China Agricultural University, Removal effects of four aquatic plants on veterinary antibiotics, 40, 67-73, (2019) 